\title{
HER2-Positive Metaplastic Spindle Cell Carcinoma Associated with Synchronous Bilateral Apocrine Carcinoma of the Breast
}

\author{
Katsumi Kito, ${ }^{1}$ Toshiharu Maeda, ${ }^{1}$ Keiko Ninomiya, ${ }^{1}$ Atsuro Sugita, ${ }^{1}$ Teiri Sagawa, \\ Kinya Matsuoka, ${ }^{2}$ Kousei Kinoshita, ${ }^{1}$ Naoki Hyodo, ${ }^{1}$ Nagisa Morita, ${ }^{1}$ and Keizo Furuya ${ }^{1}$ \\ ${ }^{1}$ Department of Pathology, Ehime Prefectural Central Hospital, 83 Kasuga-cho, Matsuyama, Ehime 790-0024, Japan \\ ${ }^{2}$ Department of Surgery, Ehime Prefectural Central Hospital, Matsuyama 790-0024, Japan
}

Correspondence should be addressed to Katsumi Kito; katsumikito@gmail.com

Received 8 July 2014; Accepted 3 September 2014; Published 18 September 2014

Academic Editor: Sami Shousha

Copyright (C) 2014 Katsumi Kito et al. This is an open access article distributed under the Creative Commons Attribution License, which permits unrestricted use, distribution, and reproduction in any medium, provided the original work is properly cited.

\begin{abstract}
Apocrine carcinoma, which is strictly defined as over $90 \%$ of tumor cells showing apocrine differentiation, is a rare variant of breast cancer. Here we report an uncommon case in which apocrine carcinomas developed concurrently in both breasts; in addition, a sarcomatoid spindle cell lesion was coincident in the right breast. Both apocrine carcinomas were immunohistochemically negative for estrogen receptor (ER) and progesterone receptor (PgR), but diffusely positive for androgen receptor (AR), GCDFP-15, and HER2. The presence of intraductal components in bilateral carcinomas and the absence of lymph node metastasis suggested that they were more likely to be individual primary lesions rather than metastatic disease. The spindle cell lesion showed a relatively well-circumscribed nodule contiguous with the apocrine carcinoma. HER2 oncoprotein overexpression was observed not only in the apocrine carcinoma, but also in the spindle cell lesion. Since the spindle cell component was intimately admixed with apocrine carcinoma and had focal cytokeratin expression, we diagnosed it as metaplastic spindle cell carcinoma, which was originated from the apocrine carcinoma. To our knowledge, this is the first case report of a patient with synchronous bilateral apocrine carcinomas coinciding with metaplastic carcinoma.
\end{abstract}

\section{Introduction}

The incidence of strictly defined apocrine carcinoma is less than $1 \%$ of all breast cancer cases, although focal apocrine differentiation is frequently observed in usual breast cancers. The criteria proposed for the diagnosis of apocrine carcinoma are more than $90 \%$ of tumor cells exhibiting apocrine features [1], characterized by large cells with sharply defined borders, abundant eosinophilic granular cytoplasm, and accumulation of secretory granules in the apical cytoplasm, the so-called apocrine snout. Immunohistochemically, apocrine carcinomas tend to be ER- and PgR-negative and AR-positive and to extensively express GCDFP-15 [1-3].

Metaplastic carcinoma is a group of neoplasms characterized by differentiation of the neoplastic epithelium into squamous cells and/or mesenchymal-looking elements [4]. Metaplastic carcinomas with mesenchymal features account for approximately $1 \%$ of all invasive carcinomas. Since synchronous bilateral apocrine carcinomas are themselves very uncommon [5], the coincidence with metaplastic carcinomas is extremely rare.

\section{Case Report}

A 52-year-old female presented with a right breast lump of 1month duration. Nipple erosion and bloody discharge were noticed prior to administration. There was no family history of breast or ovarian cancer. Physical examination revealed a firm mass $28 \mathrm{~mm}$ in size in the right breast (Figure 1). A mass of $15 \mathrm{~mm}$ in the left breast was also disclosed. Preoperative fine-needle aspiration cytology was suggestive of ductal carcinoma. No evidence of distant metastasis was observed by dynamic CT. The patient underwent bilateral total mastectomy and sentinel lymph node resection. Adjuvant chemotherapy treatment was performed after surgery, and the postoperative course was uneventful. To date, there has been no evidence of recurrence two years after surgery. 


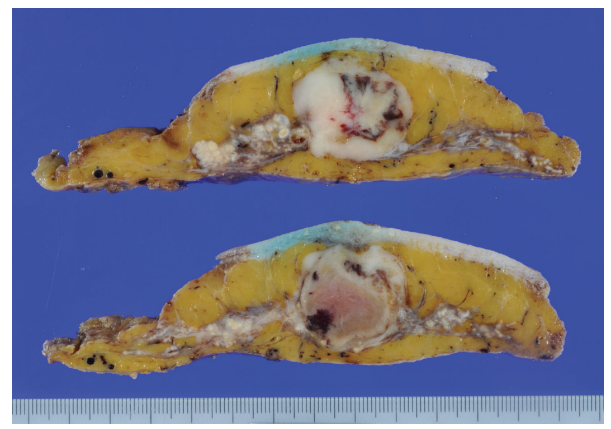

FIGURE 1: Gross appearance of the right breast tumor. The cut surface showed a relatively well-circumscribed nodule with a whitish tan color, representing metaplastic spindle cell carcinoma. The nodule was contiguous with the apocrine carcinoma component.

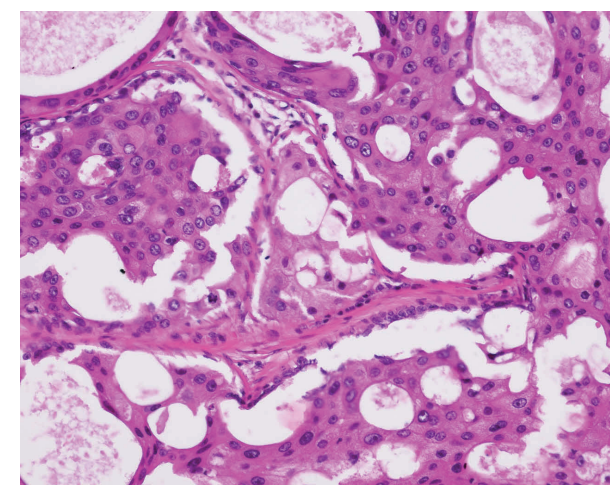

(a)

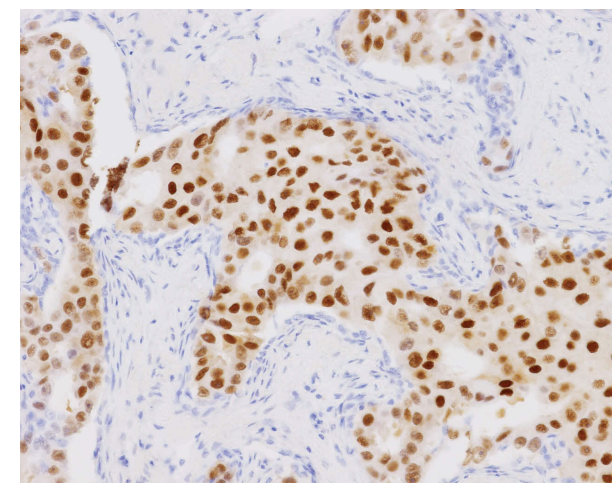

(c)

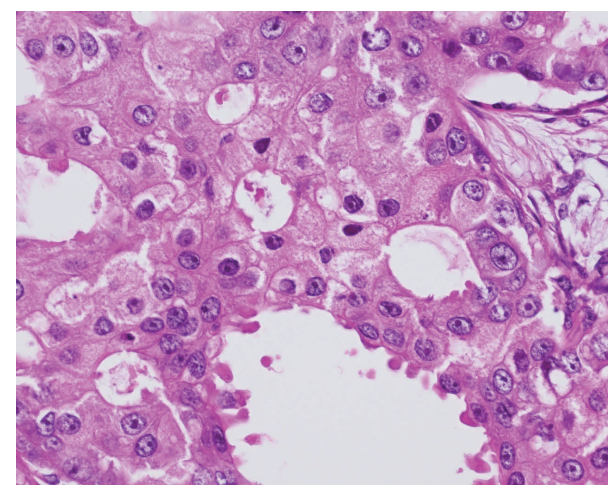

(b)

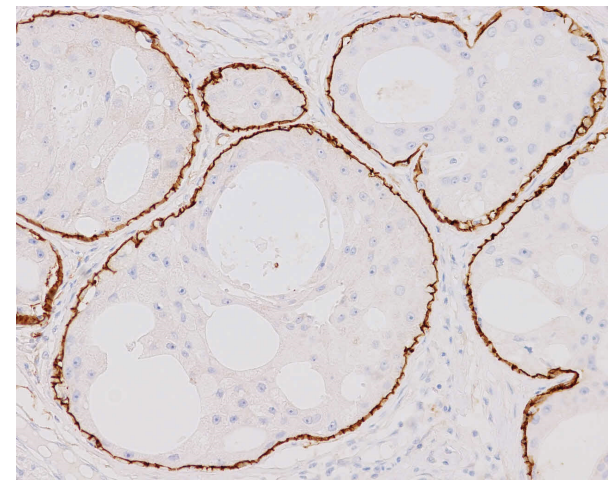

(d)

FIGURE 2: Histological features of apocrine carcinoma in the left breast. ((a) and (b)) HE. The tumor cells had abundant eosinophilic cytoplasm and apocrine snouts. (c) Tumor cells expressed androgen receptors in their nuclei. (d) The intraductal component is represented by a continuous myoepithelial cell layer detected by CD10 staining.

\section{Pathological Findings}

Pathological examination revealed that bilateral breast tumors were invasive carcinoma demonstrating extensive apocrine features. The tumor cells were polygonal in shape with well-defined cell borders and contained abundant eosinophilic cytoplasm. The tumors were composed of compact nests and intraductal components. Apocrine snouts were also observed. Immunohistochemistry demonstrated that the bilateral tumors were consistently negative for ER and PgR, but positive for AR, GCDFP-15, and HER2. These definite apocrine features were observed in over $90 \%$ of the lesions in both breasts. Intraductal components showing apocrine features, where a continuous myoepithelial cell layer was confirmed by p63 and CD10 immunostaining, were observed in both breasts and were especially predominant in the left (Figure 2). Comedo-type necrosis was observed in the intraductal lesions. Eczema-like eroded lesion of the right nipple was attributed to intraepidermal spread of the apocrine carcinoma. The apocrine carcinoma extended 


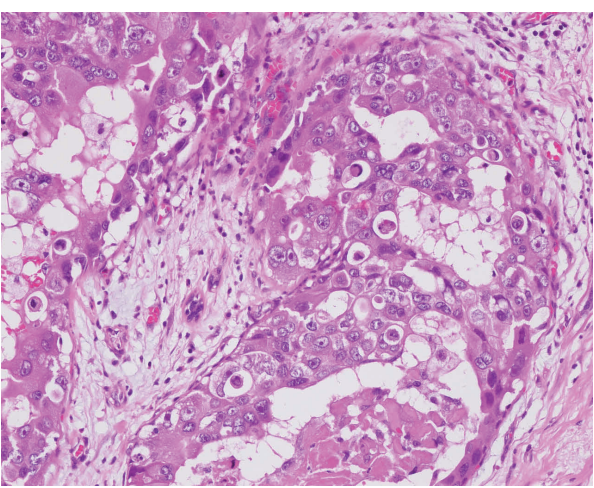

(a)

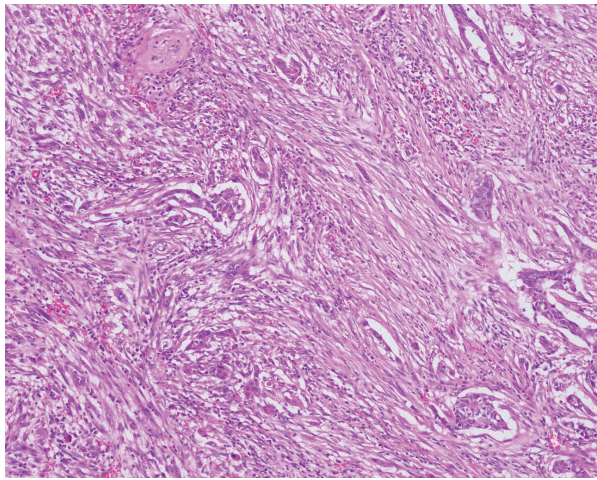

(c)

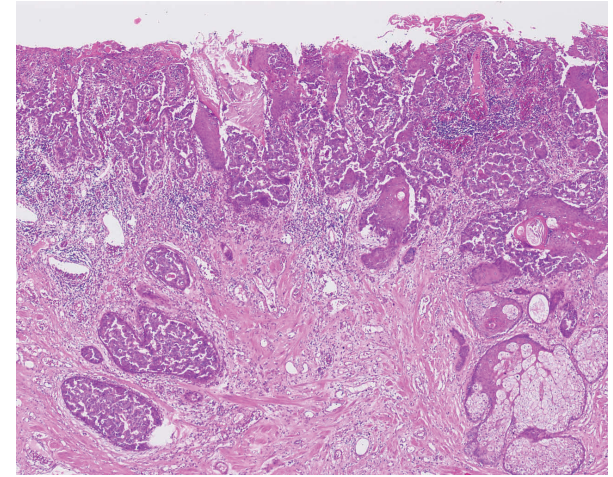

(b)

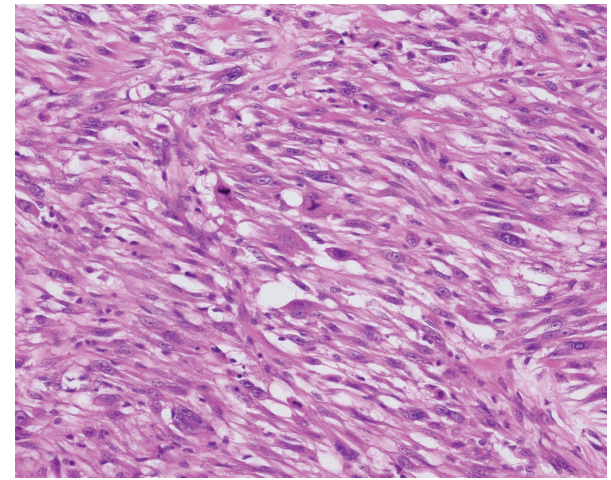

(d)

FIGURE 3: Histological features of apocrine and metaplastic carcinomas in the right breast. (a) HE. Apocrine carcinoma including intraductal components. (b) Intraepithelial invasion in nipple erosion had a Pagetoid appearance. (c) The apocrine carcinoma was admixed with the sarcomatoid spindle cell lesion. (d) The spindle cell lesion showed high nuclear polymorphism and atypia. Atypical mitotic figures are seen.

into the subareolar ducts and demonstrated a Pagetoid appearance. Neither vascular invasion nor metastasis in sentinel nodes was seen.

In addition, the right breast harbored a relatively wellcircumscribed firm nodule which was a whitish tan in color and measured $30 \mathrm{~mm}$. The nodule was totally composed of fibroblast-like spindle cells interspersed with varying collagen bundles. The spindle cells were arranged in fascicles, occasionally in storiform pattern, and the nuclei were pleomorphic with coarse chromatin and a significant number of mitotic figures (Figure 3). The spindle cell lesion contained bizarre multinucleated-giant cells and coagulative necrosis. The spindle cell lesion was contiguous with the apocrine carcinoma and a mixture of both components was observed at the periphery. The leaf-like pattern of phyllodes tumors was not observed.

Immunohistochemically, the spindle cell lesion was diffusely positive for vimentin and CD10 and focally positive for alpha-smooth muscle actin. A small number of spindle cells were positive for cytokeratin CAM5.2, whereas most CKs (AE1/AE3, 34 $\beta E 12$, and CK5/6) and p63 were negative. The spindle cells were also constantly negative for ER, PgR, and AR. Intriguingly, extensive membranous immunoreactivity for HER2 was observed in the spindle cells as well as the apocrine carcinomas (Figure 4). The immunohistochemical results are listed in Table 1.

\section{Discussion}

The prevalence of synchronous bilateral breast cancer is less than $2 \%$ of all breast cancers [6]; however, the definition of synchronous multiple cancers is somewhat unclear in the literature, especially in terms of time interval. In general, a new primary carcinoma diagnosed within 2 months is considered synchronous, whereas 12 months would seem more appropriate from an epidemiological point of view [79]. It has been recognized that synchronous bilateral breast tumors most often represent genomically individual primary tumors $[10,11]$.

It is a debatable issue whether bilateral breast carcinomas are multicentric or metastatic lesions, especially when their histological subtypes are identical. Although different histological subtypes in bilateral breast cancers suggest their multiplicity, it has been documented that synchronous bilateral invasive carcinomas have a high concordance of histological subtypes [12]. It is conceivable that the similarities between the two tumors can be explained by the fact that they develop from the same genetic background and in the same hormonal and environmental status. 


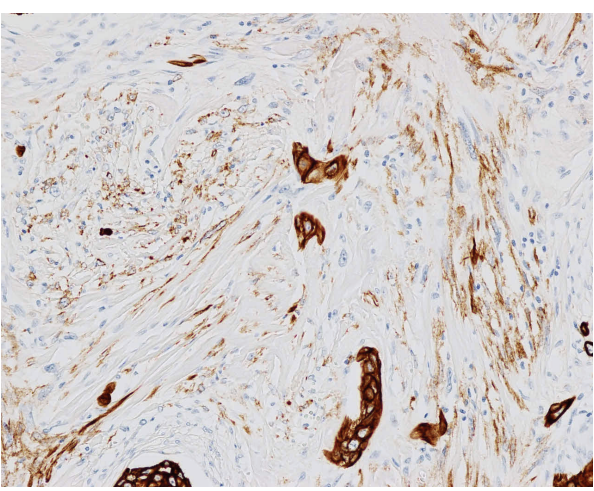

(a)

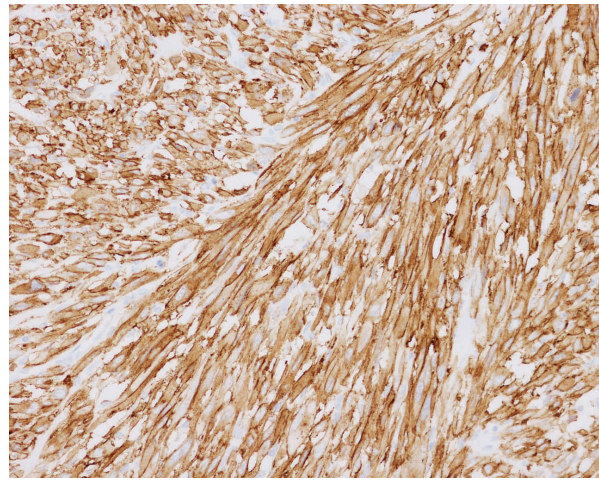

(c)

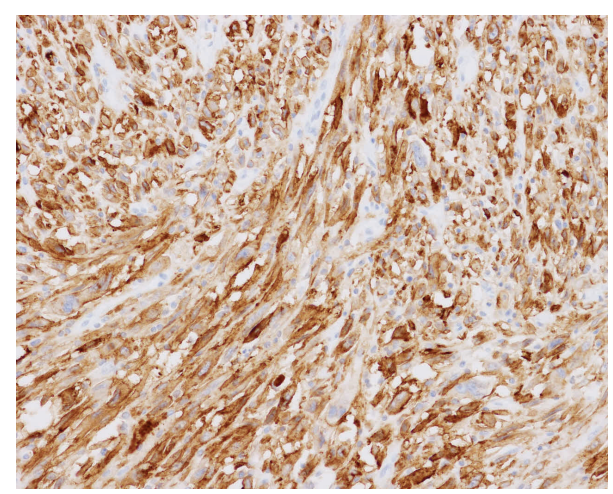

(b)

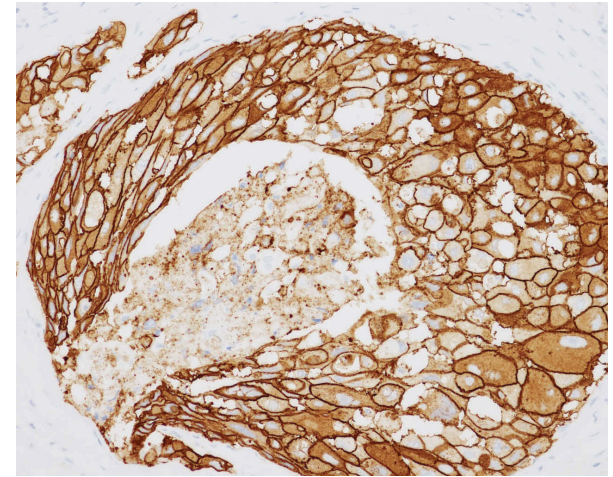

(d)

FIGURE 4: Immunohistochemical staining of the right breast carcinoma. (a) Spindle-shaped tumor cells were focally and weakly positive for cytokeratin CAM5.2, but diffusely and strongly positive for (b) CD10 and (c) HER2 oncoprotein. (d) Apocrine carcinomas strongly expressed HER2 in their cell membrane.

TABLE 1: Immunohistochemical results for each tumor morphology.

\begin{tabular}{lccc}
\hline Immunohistochemistry & Left apocrine & Right apocrine & Spindle \\
\hline ER & - & - & - \\
PgR & - & - & - \\
AR & + & + & - \\
GCDFP-15 & + & + & - \\
HER2 & + & + & + \\
Cytokeratin CAM5.2 & + & + & Focal \\
Cytokeratin AE1/AE3 & + & + & - \\
Cytokeratin 34ßE12 & Focal & Focal & - \\
Cytokeratin 5/6 & - & - & - \\
Vimentin & - & - & + \\
CD10 & - & - & + \\
$\alpha$-Smooth muscle actin & - & - & Focal \\
h-Caldesmon & - & - & - \\
Bcl-2 & - & - & - \\
CD34 & - & - & - \\
p63 & - & - & - \\
MIB-1 index & $13.0 \%$ & $10.5 \%$ & $37.3 \%$
\end{tabular}

+: positive; -: negative.

The presence of intraductal carcinoma supports the diagnosis of primary breast cancer [13]. In the present case, a significant amount of intraductal components showing apocrine features was observed in both breasts. The existence of intraductal components bilaterally strongly suggested the independent rather than metastatic nature of these lesions. Molecular analysis has demonstrated that synchronous unilateral tumor pairs are often genomically similar, whereas synchronous bilateral tumors are most likely to be nonidentical $[10,14]$. However, recent molecular analysis using microarray-based comparative genomic hybridization demonstrated an exceptional case in which even bilateral tumors containing an intraductal component nevertheless seemed most likely to be contralateral tumor spread [10]. Thus, extensive molecular analysis may be needed to conclude whether any two histologically identical breast tumors are intrinsically independent.

Sarcomatoid spindle cell carcinomas can be differentiated from true sarcomas such as malignant phyllodes tumors or periductal stromal sarcomas. It has been proposed that NOStype sarcoma with CD10 expression arises from stem cells and differentiates to myoepithelial cells to some degree, but the histogenesis remains uncertain [15]. Recent studies have suggested that the immunophenotype with CD10 expression represents a myoepithelial feature, and CD10 is one of the useful markers to track stem cells in breast carcinomas, especially precursors to sarcomatoid metaplastic carcinomas [16]. Since some NOS-type sarcoma with CD10 expression 
and most metaplastic carcinoma show positivity for $\alpha$-SMA and p63, differential diagnosis can be extremely difficult. Several antibodies are useful in differential diagnosis between metaplastic carcinoma and true sarcoma, although there is no consensus on the minimal antibody panel. The expression of CD34 and Bcl-2, which is generally seen in malignant phyllodes tumors and periductal stromal sarcomas, has not been observed in this case. Moreover, the fact that the spindle cell components were intimately admixed with apocrine carcinoma and were focally positive for CAM5.2 strongly suggested the possibility of metaplastic carcinoma rather than sarcomas [4]. It should be noted that the expression of cytokeratins in metaplastic carcinomas is frequently focal.

It has been reported that metaplastic carcinomas are consistently negative for ER and PgR and generally do not overexpress HER2 [17]; however, the present case maintained extensive HER2 positivity not only in the apocrine carcinoma including the intraductal components but also in the spindle cell lesion. Since the expression of HER2 by the spindle cell component is unusual, the consistent HER2 overexpression in the spindle cell carcinoma strongly suggested its origin in the apocrine carcinoma. Although HER2-positive breast cancers tend to behave more aggressively, high HER2 expression meets the eligibility for anti-HER2 antibody therapy.

In summary, we present a very rare case of HER2positive metaplastic spindle cell carcinoma coinciding with synchronous bilateral apocrine carcinomas.

\section{Conflict of Interests}

The authors declare that there is no conflict of interests regarding the publication of this paper.

\section{References}

[1] F. A. Tavassoli and V. Eusebi, AFIP Atlas of Tumor Parhology, Fourth Series Fascicle 10 Tumors of the Mammary Gland, Armed Forces Institute of Pathology, Washington, DC, USA, 2009.

[2] Z. Gatalica, "Immunohistochemical analysis of apocrine breast lesions: consistent over-expression of androgen receptor accompanied by the loss of estrogen and progesterone receptors in apocrine metaplasia and apocrine carcinoma in situ," Pathology Research and Practice, vol. 193, no. 11-12, pp. 753-758, 1997.

[3] Y. Tsutsumi, "Apocrine carcinoma as triple-negative breast cancer: novel definition of apocrine-type carcinoma as estrogen/progesterone receptor-negative and androgen receptorpositive invasive ductal carcinoma," Japanese Journal of Clinical Oncology, vol. 42, no. 5, pp. 375-386, 2012.

[4] J. S. Reis-Fiho, S. R. Lakhani, H. Gobbi, and N. Sneige, "Metaplastic carcinoma," in World Health Organization Classification of Tumours of the Breast, S. R. Lakhani, I. O. Ellis, S. J. Schnitt, P. H. Tan, and M. J. van de Vijver, Eds., pp. 48-52, IARC Press, Lyon, France, 2012.

[5] F. C. Schmitt, R. Soares, and R. Seruca, "Bilateral apocrine carcinoma of the breast: molecular and immunocytochemical evidence for two independent primary tumours," Virchows Archiv, vol. 433, no. 6, pp. 505-509, 1998.
[6] J. Kelmendi de Ustaran and R. P. Meiss, "Primary synchronous bilateral breast cancer: epidemiological approach," Breast Cancer Research and Treatment, vol. 12, no. 3, pp. 311-314, 1988.

[7] D. E. Heron, L. T. Komarnicky, T. Hyslop, G. F. Schwartz, and C. M. Mansfield, "Bilateral breast carcinoma: risk factors and outcomes for patients with synchronous and metachronous disease," Cancer, vol. 88, no. 12, pp. 2739-2750, 2000.

[8] H. L. Howe, R. Weinstein, R. Alvi, B. Kohler, and J. H. Ellison, "Women with multiple primary breast cancers diagnosed within a five year period, 1994-1998," Breast Cancer Research and Treatment, vol. 90, no. 3, pp. 223-232, 2005.

[9] M. Hartman, K. Czene, M. Reilly et al., "Incidence and prognosis of synchronous and metachronous bilateral breast cancer," Journal of Clinical Oncology, vol. 25, no. 27, pp. 4210-4216, 2007.

[10] S. Brommesson, G. Jönsson, C. Strand et al., "Tiling array-CGH for the assessment of genomic similarities among synchronous unilateral and bilateral invasive breast cancer tumor pairs," BMC Clinical Pathology, vol. 8, no. 1, article 6, 2008.

[11] E. N. Imyanitov, E. N. Suspitsin, M. Y. Grigoriev et al., "Concordance of allelic imbalance profiles in synchronous and metachronous bilateral breast carcinomas," International Journal of Cancer, vol. 100, no. 5, pp. 557-564, 2002.

[12] D. Huo, S. Melkonian, P. J. Rathouz, A. Khramtsov, and O. I. Olopade, "Concordance in histological and biological parameters between first and second primary breast cancers," Cancer, vol. 117, no. 5, pp. 907-915, 2011.

[13] P. J. Dawson, "What is new in our understanding of multifocal breast cancer," Pathology Research and Practice, vol. 189, no. 1, pp. 111-116, 1993.

[14] M. R. Teixeira, F. R. Ribeiro, L. Torres et al., "Assessment of clonal relationships in ipsilateral and bilateral multiple breast carcinomas by comparative genomic hybridisation and hierarchical clustering analysis," British Journal of Cancer, vol. 91, no. 4, pp. 775-782, 2004.

[15] S. Leibl and F. Moinfar, "Mammary NOS-type sarcoma with CD10 expression: a rare entity with features of myoepithelial differentiation," The American Journal of Surgical Pathology, vol. 30, no. 4, pp. 450-456, 2006.

[16] E. Bachelard-Cascales, M. Chapellier, E. Delay et al., "The CD10 enzyme is a key player to identify and regulate human mammary stem cells," Stem Cells, vol. 28, no. 6, pp. 1081-1088, 2010.

[17] J. D. Beatty, M. Atwood, R. Tickman, and M. Reiner, "Metaplastic breast cancer: clinical significance," The American Journal of Surgery, vol. 191, no. 5, pp. 657-664, 2006. 


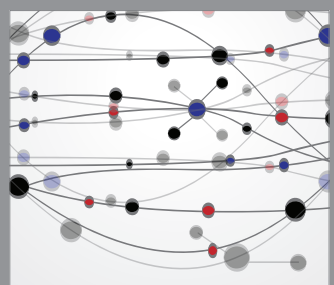

The Scientific World Journal
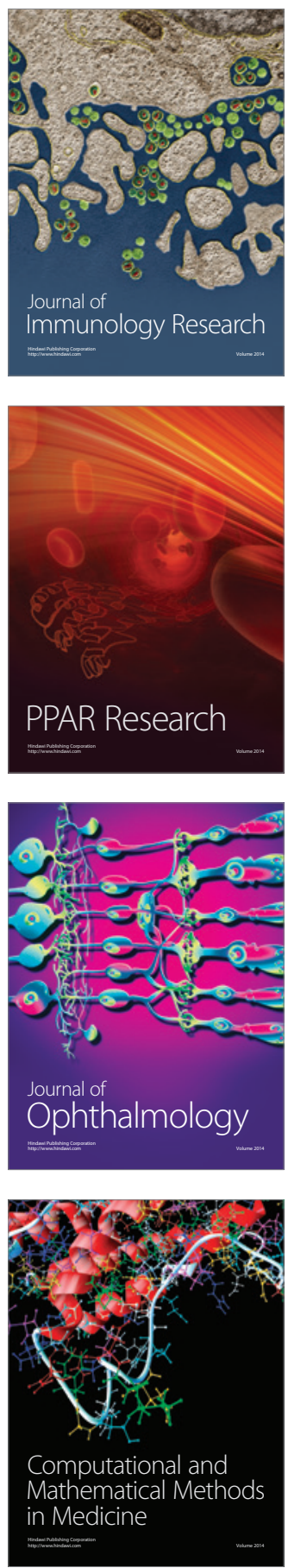

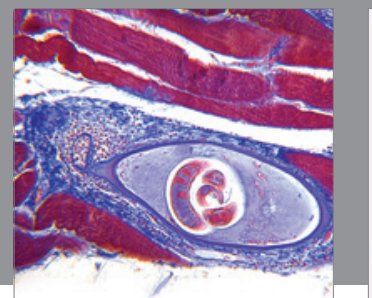

Gastroenterology

Research and Practice
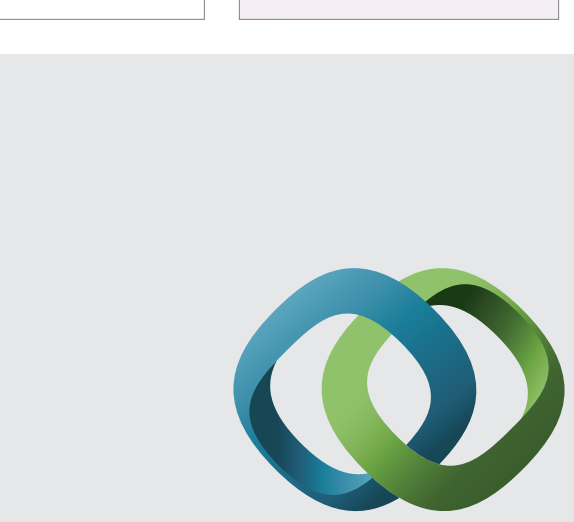

\section{Hindawi}

Submit your manuscripts at

http://www.hindawi.com
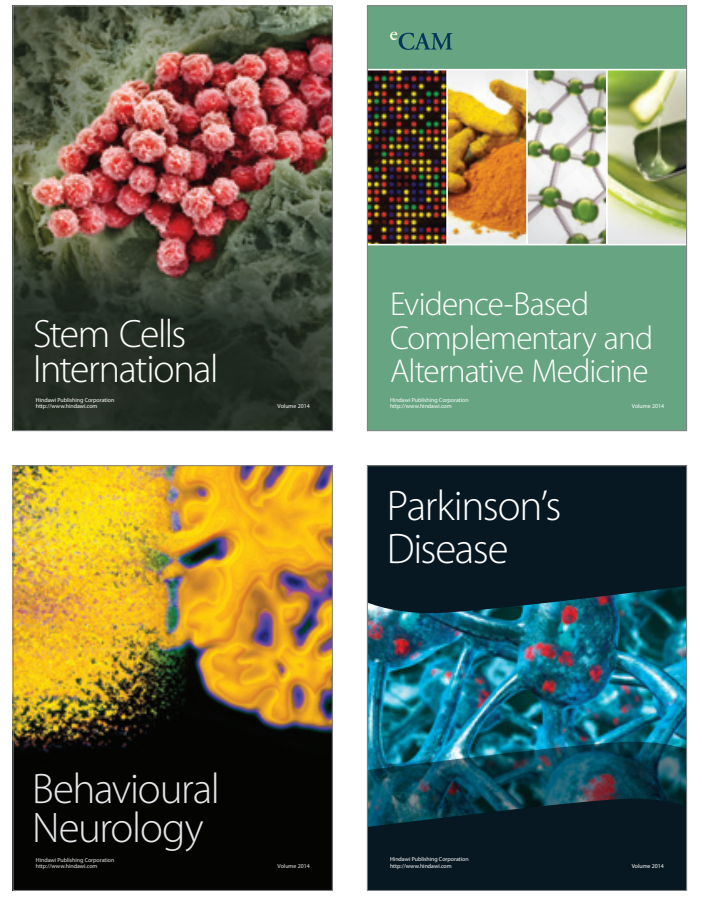
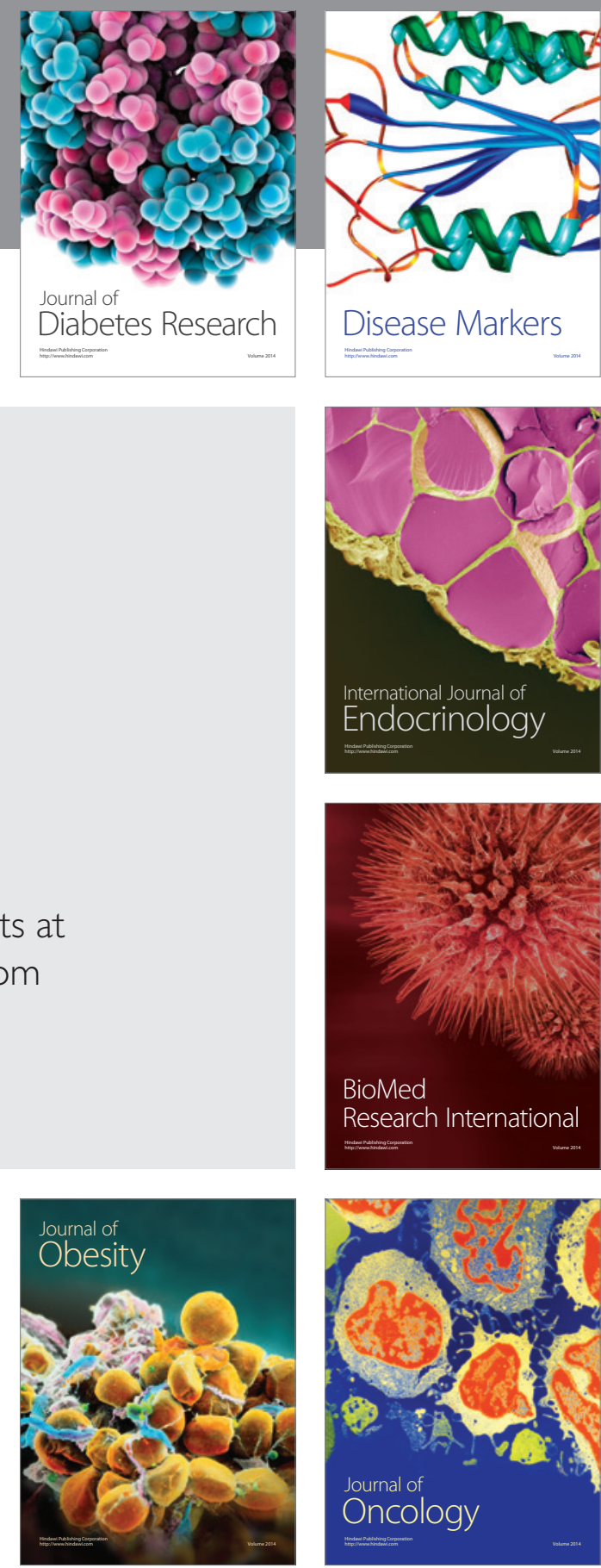

Disease Markers
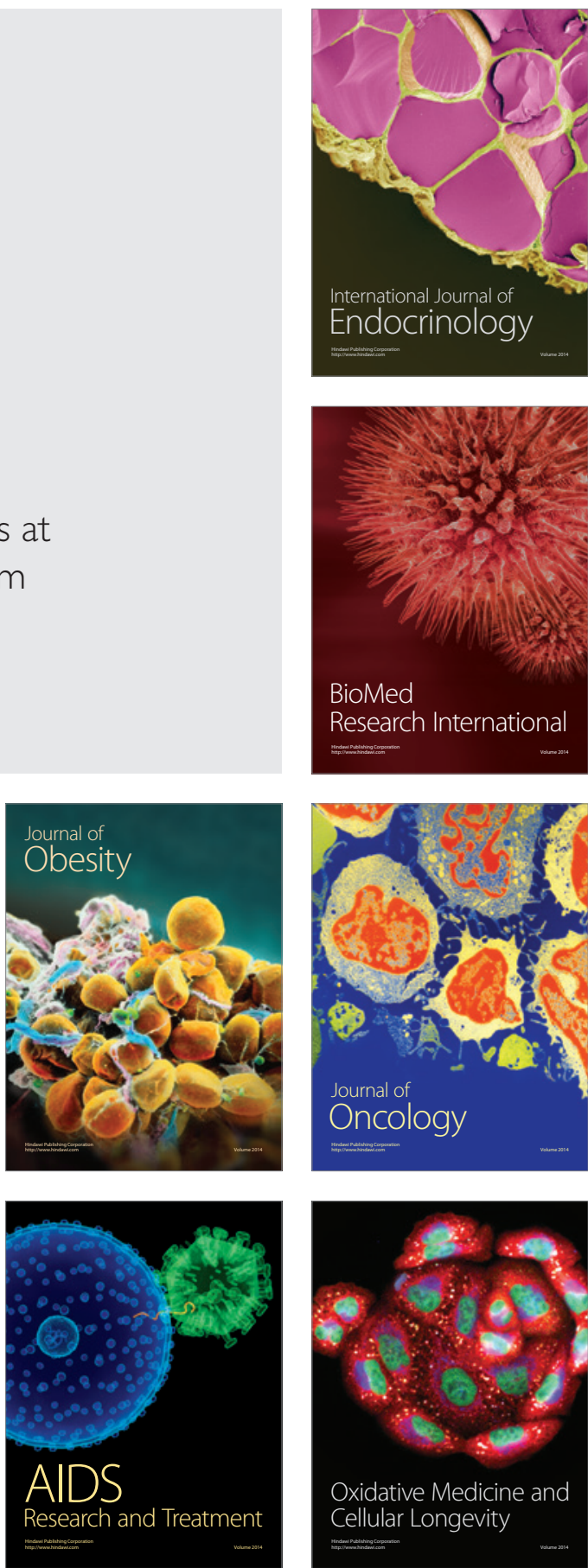